\title{
Rethinking serializable multiversion concurrency control
}

\author{
Jose M. Faleiro \\ Yale University \\ jose.faleiro@yale.edu
}

\author{
Daniel J. Abadi \\ Yale University \\ dna@cs.yale.edu
}

\begin{abstract}
Multi-versioned database systems have the potential to significantly increase the amount of concurrency in transaction processing because they can avoid read-write conflicts. Unfortunately, the increase in concurrency usually comes at the cost of transaction serializability. If a database user requests full serializability, modern multi-versioned systems significantly constrain read-write concurrency among conflicting transactions and employ expensive synchronization patterns in their design. In main-memory multi-core settings, these additional constraints are so burdensome that multiversioned systems are often significantly outperformed by singleversion systems.

We propose BoHM, a new concurrency control protocol for mainmemory multi-versioned database systems. BoHM guarantees serializable execution while ensuring that reads never block writes. In addition, BOHM does not require reads to perform any bookkeeping whatsoever, thereby avoiding the overhead of tracking reads via contended writes to shared memory. This leads to excellent scalability and performance in multi-core settings. BOHM has all the above characteristics without performing validation based concurrency control. Instead, it is pessimistic, and is therefore not prone to excessive aborts in the presence of contention. An experimental evaluation shows that BOHM performs well in both high contention and low contention settings, and is able to dramatically outperform state-of-the-art multi-versioned systems despite maintaining the full set of serializability guarantees.
\end{abstract}

\section{INTRODUCTION}

Database systems must choose between two alternatives for handling record updates: (1) overwrite the old data with the new data ("update-in-place systems") or (2) write a new copy of the record with the new data, and delete or reduce the visibility of the old record ("multi-versioned systems"). The primary advantage of multiversioned systems is that transactions that write to a particular record can proceed in parallel with transactions that read the same record; read transactions do not block write transactions since they can read older versions until the write transaction has committed. On the other hand, multi-versioned systems must consume additional space to store the extra versions, and incurs additional complexity to maintain them. As space becomes increasingly cheap in modern hardware configurations, the balance is shifting, and the majority of recently architected database systems are choosing the multiversioned approach.

This work is licensed under the Creative Commons AttributionNonCommercial-NoDerivs 3.0 Unported License. To view a copy of this license, visit http://creativecommons.org/licenses/by-nc-nd/3.0/. Obtain permission prior to any use beyond those covered by the license. Contact copyright holder by emailing info@vldb.org. Articles from this volume were invited to present their results at the 41st International Conference on Very Large Data Bases, August 31st - September 4th 2015, Kohala Coast, Hawaii.

Proceedings of the VLDB Endowment, Vol. 8, No. 11

Copyright 2015 VLDB Endowment 2150-8097/15/07.
While concurrency control techniques that guarantee serializability in database systems that use locking to preclude write-write and read-write conflicts are well understood, it is much harder to guarantee serializability in multi-versioned systems that enable reads and writes of the same record to occur concurrently. One popular option that achieves a level of isolation very close to full serializability is "snapshot isolation" [6]. Snapshot isolation guarantees that each transaction, $T$, reads the database state resulting from all transactions that committed before $T$ began, while also guaranteeing that $T$ is isolated from updates produced by transactions that run concurrently with $T$. Snapshot isolation comes very close to fully guaranteeing serializability, and indeed, highly successful commercial database systems (such as older versions of Oracle) implement snapshot isolation when the user requests the "serializable" isolation level [16]. However, snapshot isolation is vulnerable to serializability violations $[6,13]$. For instance, the famous write-skew anomaly can occur when two transactions have an overlapping read-set and disjoint write-set, where the write-set (of each transaction) includes elements from the shared read-set [6]. Processing such transactions using snapshot isolation can result in a final state that cannot be produced if the transactions are processed serially.

There has been a significant amount of work on making multiversioned systems serializable, either by avoiding the write-skew anomaly in snapshot isolation systems $[11,12]$, or by using alternative concurrency control protocols to snapshot isolation $[8$, 19]. However, these solutions either severely restrict concurrency in the presence of read-write conflicts (to the extent that they offer almost no additional logical concurrency as compared to singleversioned systems) or they require more coordination and bookkeeping, which results in poorer performance in main-memory multicore settings (Section 2).

In this paper, we start from scratch, and propose BoHM, a new concurrency control protocol for multi-versioned database systems. The key insight behind Вонм is that the complexity of determining a valid serialization order of transactions can be eliminated by separating concurrency control and version management from transaction execution. Accordingly, BoHM determines the serialization order of transactions and creates versions corresponding to transactions's writes prior to their execution (Section 3). As a consequence of this design, BOHM, guarantees full serializability while ensuring that reads never block writes. Furthermore, Bонм does not require the additional coordination and book-keeping introduced by other methods for achieving serializability in multiversioned systems. The final result is perhaps the most scalable (across multiple cores) concurrency control protocol ever proposed - there is no centralized lock manager, almost all data structures are thread-local, no coordination needs to occur across threads except at the end of a large batch of transactions, and the need for latching or any kind of atomic instructions is therefore minimized (Section 3.2).

The main disadvantage of our approach is that entire transactions must be submitted to the database system before the system 
can begin to process them. Traditional cursor-oriented database access, where transactions are submitted to the database in pieces, are therefore not supported. Furthermore, the write-set of a transaction must be deducible before the transaction begins - either through explicit write-set declaration by the program that submits the transaction, or through analysis of the transaction by the database system, or through optimistic techniques that submit a transaction for a trial run to get an initial guess for its write-set, and abort the transaction if the trial run resulted in an incorrect prediction $[30,26]$.

Although these disadvantages (especially the first one) change the model by which a user submits transactions to a database system, an increasingly large number of performance sensitive applications already utilize stored-procedures to submit transactions to database systems in order to avoid paying round-trip communication costs to the database server. These applications can leverage our multi-versioned concurrency control technique without any modifications.

BOHM thus presents a new, interesting alternative in the space of multi-version concurrency control options - an extremely scalable technique, at the cost of requiring entire transactions with deducible write-sets in advance. Experiments show that BoHM achieves linear scalability up to (at least) 20 million record accesses per second with transactions being processed over dozens of cores.

In addition to contributions around multi-versioned serializability and multi-core scalability, a third important contribution of BOHM is a clean, modular design. Whereas traditional database systems use a monolithic approach, with the currency control and transaction processing components of the systems heavily cross-dependent and intertwined, BOHM completely separates these system components, with entirely separate threads performing concurrency control and transaction processing. This modular design is made possible by BOHM's philosophy of planning transaction execution in advance, so that when control is handed over to the execution threads, they can proceed without any concern for other concurrently executing transactions. This architecture greatly improves database engine code maintainability and reduces database administrator complexity.

\section{MOTIVATION}

We now discuss two fundamental issues that limit the performance of current state-of-the-art multi-version concurrency control protocols: $a$ ) the use of global counters to obtain timestamps, and b) the cost of guaranteeing serializable execution

\subsection{Centralized Timestamps}

When a multi-version database system updates the value of a record, the update creates a new version of the record. Each record may have several versions simultaneously associated with it. Multiversion databases therefore require a way to decide which of a record's versions - if any - are visible to a particular transaction. In order to determine the record visible to a transaction, the database associates timestamps with every transaction, and every version of a record.

Multi-version databases typically use a global counter to obtain unique timestamps. When a transaction needs a timestamp, the database atomically increments the value of the counter using a latch or an atomic fetch-and-increment instruction. Using a global counter to obtain timestamps works well when it is shared among a small number of physical CPU cores but does not scale to high core counts [31].

Note that the use of a global counter to assign transactions their timestamps is a pervasive design pattern in multi-version databases. The use of a global counter is not restricted to only systems which

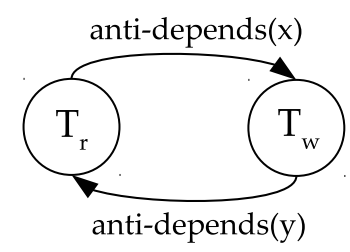

Figure 1: Non-serializable interleaving, and corresponding serialization graph of $T_{r}$ and $T_{w} . r\left[x_{1}\right]$ denotes to a read of version 1 of record $x$, correspondingly, $w\left[x_{1}\right]$ denotes a write to record $x$, which produces version 1 . A record's subscript corresponds to the version read or written by the transaction.

implement serializable isolation; implementations of weaker isolation levels such as snapshot isolation and read committed also use global counters $[8,19]$. These systems are thus subject to the scalability restrictions of using a global counter.

In order to address this bottleneck, BoHM assigns a total order to transactions prior to their execution. Each transaction is implicitly assigned a timestamp based on its position in the total order. When a transaction is eventually executed, BOHM ensures that the state of the database is identical to a serial execution of the transactions as specified by the total order. Assigning a transaction its timestamp based on its position in the total order allows BoHM to use lowoverhead mechanisms for timestamp assignment. For instance, in our implementation, we utilize a single thread which scans the total order of transactions sequentially and assigns transactions their timestamps (Section 3.2.1).

\subsection{Guaranteeing Serializability}

Multi-version database systems can execute transactions with greater concurrency than their single version counterparts. A transaction, $T_{r}$, which reads record $x$ need not block a concurrent transaction, $T_{w}$, which performs a write operation on record $x$. In order to avoid blocking $T_{w}, T_{r}$ can read a version of $x, x_{\text {old }}$ that exists prior to the version produced by $T_{w}$ 's write, $x_{n e w}$. More generally, multiversioning allows transactions with conflicting read and write sets to execute without blocking each other. Unfortunately, if conflicting transactions are processed without restraint, the resulting execution may not be serializable. In our example, if $T_{r}$ is allowed to read $x_{\text {old }}$, then it must be ordered before $T_{w}$ in the serialization order.

In the formalism of Adya et al. [1], the serialization graph corresponding to the above execution contains an anti-dependency edge from $T_{r}$ to $T_{w}$. In order for an execution of transactions to be serializable, the serialization graph corresponding to the trace of the execution cannot contain cycles. If $T_{r}$ were to write another record $y$, and $T_{w}$ read $y$ (in addition to $T_{r}$ 's read of $x$ and $T_{w}$ 's write of $x$ ), then the order of $T_{r}$ and $T_{w}$ 's operations on record $y$ must be the same as the order of their operations on record $x$. In particular, $T_{w}$ must not read $y_{\text {old }}$ (the version of $y$ prior to $T_{r}$ 's write), otherwise, the serialization graph would contain an anti-dependency edge from $T_{w}$ to $T_{r}$, leading to a cycle in the resulting serialization graph.

Figure 1 shows the the interleaved execution of transactions $T_{r}$ and $T_{w}$, and the corresponding serialization graph. The graph contains two anti-dependency edges, one from $T_{r}$ to $T_{w}$, and the other from $T_{w}$ to $T_{r}$; these two edges form a cycle, implying that the interleaving of $T_{w}$ and $T_{r}$ as described above is not serializable. This example is a variant of Snapshot Isolation's well known write-skew anomaly [6].

In order to avoid non-serializable executions such as the one described above, multi-versioned database systems need to account 
for anti-dependencies among transactions whose read and write sets conflict. There exist two ways of accounting for anti-dependencies:

- Track Reads. Whenever a transaction reads a record, the system tracks the fact that the transaction performed the read by associating some meta-data with each record in the database. The read meta-data associated with records in the database system is then used to decide on the order of transactions. For instance, the pessimistic version of Hekaton's multi-version concurrency control algorithm associates a counter with every record in the database [19]. The counter reflects the number of in-flight transactions that have read the record. As another example, Cahill et al. modify BerkeleyDB's lock manager to track anti-dependency edges to and from a particular transaction [8].

- Validate Reads. A transaction locally keeps track of the version of each record it observed. When the transaction is ready to commit, it validates that the reads it observed are consistent with a serial order. This technique is used by Hekaton's optimistic concurrency control protocol [19], and Multi-version General Validation [2].

While both approaches ensure that all executions are serializable, they come at a cost. Concurrency control protocols track reads in order to constrain the execution of concurrent readers and writers. For instance, Hekaton's pessimistic concurrency control protocol does not allow a writer to commit until all concurrent readers have either committed or aborted [19]. In addition to the reduction in concurrency resulting from the concurrency control protocol itself, tracking reads entails writes to shared memory. If a record is popular, then many different threads may attempt to update the same memory words concurrently, leading to contention for access to internal data structures, and subsequent cache coherence slowdowns. Since reads are tracked, this contention is present even if the workload is read-only.

The "Validate Reads" approach does not suffer from the problem of requiring reads to write internal data to shared memory. However, validation protocols reduce concurrency among readers and writers by aborting readers. Such a situation runs counter to the original intention of multi-version concurrency control, because allowing multiple versions of a record is supposed to allow for greater concurrency among readers and writers.

In order to address these limitations, we designed BoHM's concurrency control protocol with the following goals in mind: (1) A transaction, $T_{r}$, which reads the value of a particular record should never block or abort a concurrent transaction that writes the same record. This should be true whether or not $T_{r}$ is a read-only transaction. (2) Reading the value of a record should not require any writes to shared memory.

\section{DESIGN}

BoHM's design philosophy is to eliminate or reduce coordination among database threads due to synchronization based on writes to shared memory. BoHM ensures that threads either make decisions based on local state, or amortize the cost of coordination across several transactions. BOHM achieves this goal by separating concurrency control logic from transaction execution logic. This separation is reflected in BOHM's architecture: a transaction is processed by two different sets of threads in two phases: (1) a concurrency control phase which determines the proper serialization order and creates a data structure that will enable the second phase to process transactions without concern for concurrently executing transactions, and (2) an execution phase, which actually executes transaction's logic.
While the separation of concurrency control logic and transaction execution logic allows BOHM to improve concurrency and avoid scalability bottlenecks, it comes at the cost of extra requirements. In order to plan execution correctly, the concurrency control phase needs advance knowledge of each transaction's write-set. This requirement is not unique to BOHM - several prior systems exploit a priori information about transactions's read- and/or writesets $[3,10,30,23]$. These previous systems have shown that even though they need transactions' write- (and sometimes also read-) sets in advance, it is not necessary for transactions to pre-declare these read-/write-sets. For example, Calvin proposes a speculative technique which predicts each transaction's read-/write-sets on the fly [30]. Furthermore, Ren et. al. show that aborts due to speculative read/write-set prediction are rare, since the volatility of data used to derive the read and write sets is usually low ${ }^{1}$ [26]. BOHM can make use of this technique if transactions' write-sets are not available (or derivable) in advance. However, either way, there is a requirement that the entire transaction be submitted to the system at once. Thus, cursor-oriented transaction models that a submit a transaction to the system in pieces cannot be supported.

\subsection{System Overview}

Transactions that enter the system are handed over to a single thread which creates a log in shared-memory containing a list of all transactions that have been input to the system. The position of a transaction in this log is its timestamp. The log is read (in parallel) by $m$ concurrency control threads. These threads own a logical partition of the records in the database. For each transaction in the log, each concurrency control thread analyzes the write-set of the transaction to see if it will write any records in the partition owned by that thread. If so, the thread will create space (a "placeholder") for the new version in the database (the contents remain uninitialized) and link it to the placeholder associated with the previous version of record (which was written by the same thread).

A separate set of $n$ threads (the "transaction execution threads") read the same log of input transactions and perform the reads associated with the transactions in the $\log$ and fill in the pre-existing allocated space for any writes that they perform. These transaction execution threads do not start working on a batch of transactions until the the concurrency control threads have completed that same batch. Therefore, it is guaranteed that placeholders already exist for any writes that these threads perform. Furthermore, reads can determine which version of a record is the correct version to read (in order to guarantee serializability) by navigating the backward references of the placeholders until the record is reached which was created by a transaction older than the transaction which is performing the read and invalidated by a transaction newer than the transaction which is performing the read. If this placeholder associated the correct version to read remains uninitialized, then the read must block until the write is performed. Hence, in BOHM, reads never block writes, but writes can block reads.

The following two subsections give more details on the concurrency control phase and the transaction execution phase, respectively. Furthermore, they explain how our design upholds our philosophy of not allowing contented writes to shared memory, nor any thread synchronization at the record or transaction granularity.

\subsection{Concurrency Control}

The concurrency control layer is responsible for (1) determining the serialization order of transactions, and (2) creating a safe envi-

\footnotetext{
${ }^{1}$ For example, on TPC-C, no aborts due to speculative read/write set prediction are observed.
} 


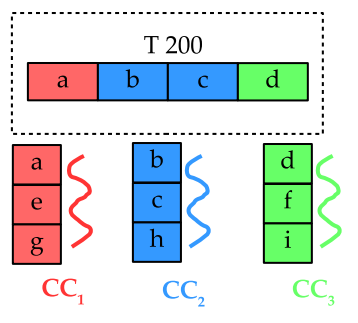

Figure 2: Intra-transaction parallelism. Transaction 200, which writes four records is shown in the upper rectangle. The logical partitioning of concurrency control thread responsibility is shown below.

ronment in which the execution phase can run transactions without concern for other transactions running concurrently.

\subsubsection{Timestamp Assignment}

The first step of the concurrency control layer is to insert each transaction into a $\log$ in main-memory. This is done by a single thread dedicated solely to this task. Because the concurrency control layer is separated from (and run prior to) transaction execution, BOHM can use this log to implicitly assign timestamps to transactions (the timestamp of a transaction is its position in the log). Since a single thread creates the log prior to all other steps in transaction processing, log creation (and thus timestamp assignment) is an uncontended operation. This distinguishes BOHM from other multi-versioned schemes that assign timestamps (which involve updating a shared counter) as part of transaction processing. Thus, timestamp assignment is an example of our design philosophy of avoiding writing and reading from shared data-structures as much as possible.

Several prior multi-version concurrency control mechanisms assign each transaction, $T$, two timestamps, $t_{\text {begin }}$ and $t_{\text {end }}[2,6,8$, 19]. $t_{\text {begin }}$ determines which versions of pre-existing records are visible to $T$, while $t_{\text {end }}$ determines the logical time at which $T$ 's writes become visible to other transactions, and is used to validate whether $T$ can commit. The time between $t_{\text {begin }}$ and $t_{\text {end }}$ determines the logical interval of time during which $T$ executes. If another transaction's logical interval overlaps with that of $T$, then the database system needs to ensure that the transactions do not conflict with each other (what exactly constitutes a conflict depends on the isolation level desired).

In contrast, BOHM assigns each transaction a single timestamp, $t s$ (determined by the transaction's position in the log). Intuitively, $t s$ "squashes" $t_{\text {begin }}$ and $t_{\text {end }}$ together; $t s$ determines both the logical time at which $T$ performs its reads, and the logical time at which $T$ 's writes are visible to other transactions. As a consequence, each transaction appears to execute atomically at time $t s$.

\subsubsection{Inserting Placeholders}

Once a transaction's timestamp has been determined, the concurrency control layer inserts a new version for every record in the transaction's write-set. This includes creating new versions for index key-values updated by the transaction. The version inserted by the concurrency control layer contains a placeholder for the value of the version, but the value is uninitialized. The actual value of the version is only produced once the corresponding transaction's logic is executed by the execution layer (Section 3.3).

Several threads contribute to the processing of a single transaction's write-set. BOHM partitions the responsibility for each record of a table across the set of concurrency control threads. When the concurrency control layer receives a transaction, every concurrency control thread examines $T$ 's write-set in order to determine whether any records belong to the partition for which it is responsible.

Figure 2 illustrates how several threads cooperatively process each transaction. The transaction is assigned a timestamp of 200, and its write-set consists of records $a, b, c$, and $d$. The concurrency control layer partitions records among three threads, $C C_{1}, C C_{2}$, and $C C_{3} . C C_{1}$ 's partition contains record $a, C C_{2}$ 's partition contains records $b$ and $c$, and $C C_{3}$ 's partition contains record $d . C C_{1}$ thus inserts a new version for record $a, C C_{2}$ does the same for records $b$ and $c$, and $C C_{3}$ for $d$. BoHM uses several threads to process a single transaction, a form of intra-transaction parallelism.

Every concurrency control thread must check whether a transaction's write-set contains records that belong to its partition. For instance, if record $d$ belonged to $C C_{1}$ 's partition instead of $C C_{3}$ 's, $C C_{3}$ would still have to check the transaction's write-set in order to determine that no records in the transaction's write-set map to its partition.

This design is consistent with our philosophy that concurrency control threads should never need to coordinate with each other in order to process a transaction. Each record is always processed by the same thread (as long as the partitioning is not adjusted); two concurrency control threads will never try to process the same record, even across transaction boundaries. The decision of which records of a transaction's write-set to process is a purely thread local decision; a concurrency control thread will process a particular record only if the record's key resides in its partition.

Not only does this lead to reduced cache coherence traffic, but it also leads to multi-core scalability. As we dedicate more concurrency control threads to processing transactions, throughput increases for two reasons. First, each transaction is processed by a greater number of concurrency control threads, which leads to an increase in intra-transaction parallelism. Since concurrency control threads do not need to coordinate with each other, there is little downside to adding additional threads as long as there are enough processing resources on which they can run. Second, for a fixed database size, the number of keys assigned to each thread's partition decreases. As a consequence, each concurrency control thread will have a smaller cache footprint.

One impediment to scalability is the fact that every concurrency control thread must examine every transaction that enters the system. This is logic which is effectively executed serially, since every concurrency control thread runs the same piece of logic. Increasing the number of concurrency control threads beyond a certain point will therefore yield a diminishing increase in throughput due to Amdahl's law. Although we have not encountered this scalability bottleneck in our experimental evaluation, a straightforward mechanism around the issue is to pre-process transactions prior to handing them over to the concurrency control layer. The pre-processing layer can analyze each transaction to determine the set of concurrency control threads responsible for the writes the transaction performs, and then forward transaction references to the appropriate concurrency control threads. Each transaction can be pre-processed independently of others, thus making the pre-processing step embarrassingly parallelizable.

\subsubsection{Processing a single transaction's read/write set}

For each record in a transaction's write-set, the concurrency control phase produces a new version to hold the transaction's write. Figure 3 shows the format of a record version. Each version consists of the following fields:

- Begin Timestamp. The timestamp of the transaction that created the record. 


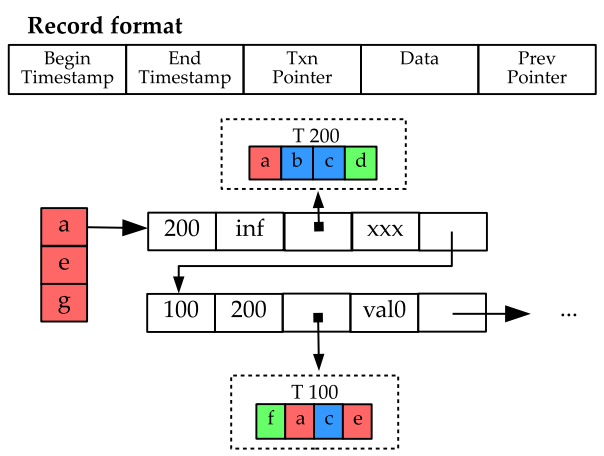

Figure 3: Inserting a new version

- End Timestamp. The timestamp of the transaction that invalidated the record.

- Txn Pointer. A reference to the transaction that must be executed in order to obtain the value of the record.

- Data. The actual value of the record.

- Prev Pointer. A reference to the version of the record that precedes the current version.

When inserting a new version of a record, the concurrency control thread sets the version's fields as follows: (1) the version's start timestamp is set to the timestamp of the transaction that creates the version, (2) the version's end timestamp is set to infinity, (3) the version's txn pointer is set to the transaction that creates the version, (4) the version's data is left uninitialized, (5) the version's prev pointer is set to the preceding version of the record.

Figure 3 shows the thread $C C_{1}$ inserting a new version of record $a$, which is produced by transaction $T_{200} . C C_{1}$ sets the new version's begin timestamp to 200, and its end timestamp to infinity. The version's txn pointer is set to $T_{200}$ (since $T_{200}$ produces the new version). At this point, the version's data has not yet been produced; BoHM needs to execute $T_{200}$ in order to obtain the value of the version.

While inserting a new version of record $a, C C_{1}$ finds that a previous version of the record exists. The older version of $a$ was produced by transaction $T_{100} . C C_{1}$ sets the new version's prev pointer to the old version, and sets the old version's end timestamp to 200 .

In order to create a new version of a record, BoHM does not need to synchronize concurrency control threads. BOHM partitions the database among concurrency control threads such that a record is always processed by the same thread, even across transaction boundaries (Section 3.2.2). One consequence of this design is that there is no contention in the concurrency control phase. The maintenance of the pointers to the current version of every record can be done in a thread-local data-structure; thus the look-up needed to populate the prev pointer in the new versions is thread-local. Furthermore, if multiple transactions update the same hot record, the corresponding new versions of the record are written by the same concurrency control thread, thereby avoiding cache coherence overhead.

For each element in the transaction's read-set, BoHM needs to identify the corresponding version that the transaction will read. In general, the concurrency control phase does not need to get involved in processing a transaction's read-set. When an execution thread that is processing a transaction with timestamp $t s$ wants to read a record in the database, it can find the correct version of the record to read by starting at the latest version of the record, and following the chain of linked versions (via the prev pointer field) until it finds a version whose $t_{\text {begin }} \leq t s$ and $t_{\text {end }} \geq t s$. If no such version exists, then the record is not visible to the transaction.

While the above-described technique to find which version of a record to read is correct, the cost of the traversal of pointers may be non-trivial if the linked list of versions is long. Such a situation may arise if a record is popular and updated often. An optimization to eliminate this cost is possible if the concurrency control phase has advanced knowledge of the read-sets of transactions (in addition to the write-set knowledge it already requires). In this case, for every record a transaction will read, concurrency control threads annotate the transaction with a reference to the correct version of the record to read. This is a low-cost operation for the concurrency control threads since the correct version is simply the most recent version at the time the concurrency control thread is running ${ }^{2}$.

In particular, if a record in a transaction's read-set resides on a concurrency control thread's logical partition, the thread looks up the latest version of the record and writes a reference to the latest version in a memory word reserved in advance within the transaction. The concurrency control thread does not track the read in the database, it merely gives the transaction a reference to the latest version of the record as of the transaction's timestamp.

A consequence of BOHM's design is that a transaction's reads do not require any contended writes to shared memory. Even for the read-set optimization mentioned above, the write containing the correct version reference for a read is to pre-allocated space for the reference within a transaction, and is uncontended since only one concurrency control thread is responsible for a particular record. In contrast, pessimistic multi-version systems such as Hekaton [19] and Serializable Snapshot Isolation [8] need to coordinate a transaction's reads with concurrent transaction's writes in order to avoid serializability violations.

\subsubsection{Batching}

Only after a transaction $T$ has been processed by all appropriate concurrency control threads can it be handed off to the transaction execution layer. One naïve way of performing this hand-off is for the concurrency control threads to notify each other after having processed each transaction by using synchronization barriers. After processing $T$, each concurrency control thread enters a global barrier in order to wait for all other threads to finish processing $T$. After all threads have entered this barrier, each concurrency control thread can begin processing the next transaction.

Unfortunately, processing transactions in this fashion is extremely inefficient. Threads need to synchronize with each other on every transaction, which has the effect of forcing concurrency control threads to effectively execute in lock step. Another issue is that some concurrency control threads are needlessly involved in this synchronization process. Consider a scenario where none of the records in $T$ 's write-set belong to a concurrency control thread, $C C$ 's, partition. $C C$ has to wait for every thread in order to move on to the next transaction despite the fact that $C C$ "contributes" nothing to $T$ 's processing.

BOHM avoids expensive global coordination on every transaction, and instead amortizes the cost of coordination across large batches of transactions. The concurrency control thread responsible for allotting each transaction a timestamp accumulates transactions in a batch. The concurrency control threads responsible for writing versions receive an ordered batch of transactions, $b$, as input. Each concurrency control thread processes every transaction in $b$ independently, without coordinating with other threads (Sections 3.2.2,

\footnotetext{
${ }^{2}$ This is true since concurrency control threads process transactions sequentially (threads derive concurrency by exploiting intratransaction parallelism).
} 
3.2.3). Once a thread has finished processing every transaction in $b$, it enters a global barrier, where it waits until all concurrency control threads have finished processing $b$, amortizing the cost of a single global barrier across every transaction in $b$.

Coordinating at the granularity of batches means that some threads may outpace others in processing a batch; a particular thread could be processing the $100^{t h}$ transaction in the batch while another is still processing the $50^{t h}$ transaction. Allowing certain concurrency control threads to outpace others is safe for the same reason that intra-transaction parallelism is safe (Section 3.2.2): Вонм partitions the database among concurrency control threads such that a particular record is always processed by the same thread, even across transaction boundaries.

\subsection{Transaction Execution}

After having gone through the concurrency control phase, a batch of transactions is handed to the transaction execution layer. The execution layer performs two main functions: it executes transactions' logic, and (optionally) incrementally garbage collects versions which are no longer visible due to more recent updates.

\subsubsection{Evaluating Transaction Logic}

The concurrency control layer inserts a new version for every record in a transaction's write-set. However, the data within the version cannot yet be read because the transaction responsible for producing the data has not yet executed; concurrency control threads merely insert placeholders for the data within each record. Each version inserted by the concurrency control layer contains a reference to the transaction that needs to be evaluated in order to obtain the data of the version.

Read Dependencies. Consider a transaction $T$, whose readset consists of $r_{1}, r_{2}, \ldots, r_{n}$. $T$ needs to read the correct version of each record in its read-set using the process described in Section 3.2.3. However, the data stored inside one or more of these correct versions may not yet have been produced because the corresponding transaction has not yet been executed. Therefore, an execution thread may not be able to complete the execution of $T$ until the transaction upon which $T$ depends has finished executing.

Write Dependencies. Every time the value of a particular record is updated, the concurrency control layer creates a new version of the record, stored separately from other versions. Consider two transactions $T_{1}$ and $T_{2}$, such that (1) neither transactions' logic contain aborts, and (2) $T_{1}$ is processed before $T_{2}$ by the concurrency control layer. Both transactions' write-sets consist of a single record, $x$, while their read-sets do not contain record $x$. In this scenario, the concurrency control layer will write out two versions corresponding to record $x$, one each for $T_{1}$ 's and $T_{2}$ 's update. The order of both transaction's updates is already decided by the concurrency control layer; therefore, $T_{1}$ and $T_{2}$ 's execution need not be coordinated. In fact, $T_{2}$ could execute before $T_{1}$, despite the fact that $T_{1}$ precedes $T_{2}$, and their write-sets overlap. However, if $T_{2}$ performs a read-modify-write of record $x$, then $T_{2}$ must wait for the version of $x$ produced by $T_{1}$ before it can proceed with the write (this is a type of read dependency explained above). If $T_{2}$ aborts, then it also needs to wait for $T_{1}$. The reason is that in this case, the data written to its version of $x$ is equal to that produced by $T_{1}$. Thus, $T_{2}$ has a read dependency on $T_{1}$.

We now describe how a set of execution threads execute a batch of transactions handed over from the concurrency control layer. The execution layer receives a batch of transactions in an ordered array $<T_{0}, T_{1}, \ldots, T_{n}>$. The transactions are partitioned among $k$ execution threads such that thread $i$ is responsible for ensuring transactions $T_{i}, T_{i+k}, T_{i+2 k}$, and so forth are processed. Thread $i$ does not need to directly execute all transactions that it is responsible for - other threads are allowed to execute transactions assigned to $i$, and $i$ is allowed to execute transactions assigned to other threads. However, before moving onto a new batch of transactions, thread $i$ must ensure that all transactions that it is responsible for in the current batch have been executed.

Each transaction can be in one of three states: Unprocessed, Executing, and Complete. All transactions received from the concurrency control layer are in state Unprocessed - this state corresponds to transactions whose logic has not yet been evaluated. A transaction is in state Executing if an execution thread is in the process of evaluating the transaction. A transaction whose logic has been evaluated is in state Complete.

In order to process a transaction, $T$, an execution thread, $E$, attempts to atomically change $T$ 's state from Unprocessed to Executing. E's attempt fails if $T$ is either already in state Executing or Complete. If $E$ 's attempt is successful, then BOHM can be sure that $E$ has exclusive access to $T$; subsequent transactions that try to change $T$ 's state from Unprocessed to Executing will fail.

If, upon trying to read a record, $E$ discovers a read dependency on a version that has yet to be produced, $E$ tries to recursively evaluate the transaction $T^{\prime}$ which must be evaluated to produce the needed version. If $E$ cannot evaluate $T^{\prime}$ (because another thread is already processing $T^{\prime}$ ) then $E$ sets $T$ 's state back to Unprocessed. $T$ is later picked up by an execution thread (not necessarily $E$ ) which attempts once again to execute the transaction. After completing all reads and writes for $T, E$ sets $T$ 's state to Complete.

Note that execution and concurrency control threads operate on different batches concurrently. Execution threads are responsible for producing the data associated with versions written in a batch, while concurrency control threads create versions and update the appropriate indexes. Logically, a version's data is a field associated with the version (Section 3.2.3, Figure 3). Execution threads only write a version's data field; therefore, there are no write-write conflicts between execution and concurrency control threads. However, in order to locate the record whose data must be read or written, execution threads may need to read database indexes. Execution threads need only coordinate with a single writer thread while reading an index - the concurrency control thread responsible for updating the index entry for that record. BOHM uses standard latchfree hash-tables to index data; readers need only spin on inconsistent or stale data [18]. We believe that coordinating structural modifications (SMOs) by a single writer with multiple readers is significantly less complex than coordinating multiple writers and readers. We leave the broader discussion of SMOs in general indexing structures to future work.

\subsubsection{Garbage Collection}

BOHM can be optionally configured to automatically garbage collect all versions that are no longer visible to any active or future transactions. Records that have been "garbage collected" can be either deleted or archived. This section describes how Вонм decides when a version can be safely garbage collected.

BoHM's execution layer receives transactions in batches. Transactions are naturally ordered across batches; if batch $b_{0}$ precedes batch $b_{1}$, then every transaction in $b_{0}$ precedes every transaction in $b_{1}$. Assume that a transaction $T$ belongs to batch $b_{0} . T$ updates the value of record $r$, whose version is $v_{1}$, and produces a new version $v_{2}$. The timestamp of any transaction in batch $b_{i}$, where $i \geq 1$, will always exceed $v_{2}, T$ 's timestamp. As a consequence, version $v_{1}$, which precedes $v_{2}$, will never be visible to transactions in batches which occur after $b_{0}$. Section 3.3.1 explained that execution threads always process batches sequentially; that is, each thread will not 
move onto batch $b_{i+1}$ until the transactions it is assigned in $b_{i}$ have been executed. Therefore, $v_{1}$ can be garbage collected when every execution thread has finished executing batch $b_{0}$. This condition holds regardless of which batch $v_{1}$ was created in. In fact, $v_{1}$ may even have been created in $b_{0}$. Whenever a transaction in batch $b_{i}$ updates the value of a particular record, we can garbage collect the preceding version of the record when every execution thread has finished processing every transaction in batch $b_{i}$.

Garbage collection based on the preceding intuition is amenable to an efficient and scalable implementation based on read-copyupdate (RCU) [21]. The heart of the technique is maintaining a global low-watermark corresponding to the minimum batch of transactions processed by every execution thread. Each execution thread $t_{i}$ maintains a globally visible variable batch $_{i}$, which corresponds to the batch most recently executed by $t_{i}$. batch $h_{i}$ is only updated by $t_{i}$. We designate one of the execution threads, $t_{0}$, with the responsibility of periodically updating a global variable lowwatermark with $\min \left(\right.$ batch $\left._{i}\right)$, for each $i$.

\section{EXPERIMENTAL EVALUATION}

BOHM's primary contribution is a multi-version concurrency control protocol that is able to achieve serializability at lower cost than previous multi-version concurrency control protocols. Therefore, the best comparison points for ВонM are other multi-versioned protocols. We thus compare BoHM's performance to two state-ofthe-art multi-versioned protocols: the optimistic variant of Hekaton [19], and Snapshot Isolation (implemented within our Hekaton codebase) [6]. Our Hekaton and Snapshot Isolation (SI) implementations include support for commit dependencies, an optimization that allows a transaction to speculatively read uncommitted data. In order to keep our codebase simple, our Hekaton and SI implementations do not incrementally garbage collect versions from the database and use a simple fixed-size array index to access records (BOHM and its other comparison points discussed below use dynamic hash-tables). The lack of garbage collection does not negatively impact performance; on the contrary, garbage collection was cited as one of the primary contributors to Hekaton's poor performance relative to single-versioned systems. BOHM runs with garbage collection enabled; therefore, any performance gains of BoHM over Hekaton and SI that we see in our experiments are conservative estimates. We would expect an even larger performance difference had garbage collection of these baselines been turned on.

While Hekaton and SI are the main points against which we seek to compare BoHM, our evaluation also includes single-version baselines. We compare Вонм against state-of-the-art optimistic concurrency control (OCC) and two-phase locking (2PL) implementations. Our OCC implementation is a direct implementation of Silo [31] - it validates transactions using decentralized timestamps and avoids all shared-memory writes for records that were only read. All our optimistic baselines - single-version OCC, Hekaton, and SI - are configured to retry transactions in the event of an abort induced by concurrency control.

Our 2PL implementation uses a hash-table to store information about the locks acquired by transactions. Our locking implementation has three important properties. a) Fine-grained latching. We use per-bucket latches on the lock table to avoid a centralized latch bottleneck. $b$ ) Deadlock freedom. We exploit advance knowledge of transactions's read- and write-sets to acquire locks in lexicographic order. Acquiring locks in this fashion is guaranteed to avoid deadlocks. Consequently, our locking implementation does not require any deadlock detection logic. c) No lock table entry allocations. We exploit advance knowledge of a transaction's read- and write-sets to allocate a sufficient number of lock table entries prior

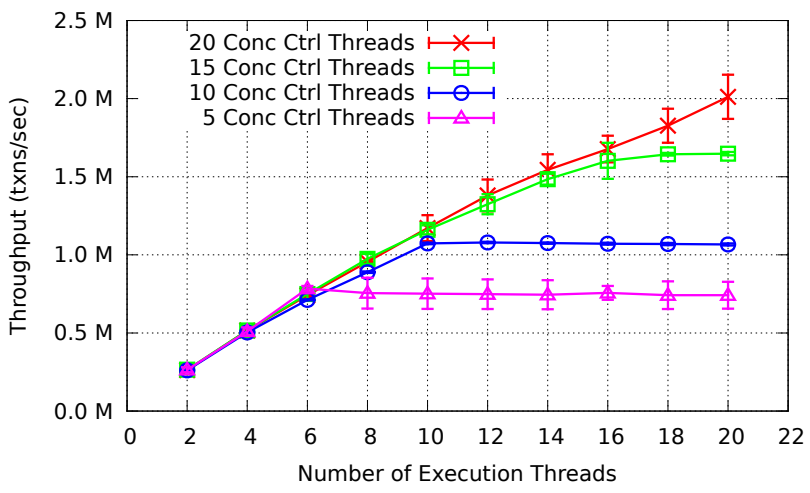

Figure 4: Interaction between concurrency control and transaction execution modules.

to submitting the transaction to the database. The consequence of this design is that the duration for which locks are held is reduced to the bare minimum.

Our experimental evaluation is conducted on a single 40 -core machine, consisting of four 10-core Intel E7-8850 processors and 128GB of memory. Our operating system is Linux 3.9.2. All experiments are performed in main-memory, so secondary storage is not utilized for our experiments.

In all our implementations, there is a $1: 1$ correspondence between threads and cpu cores; we explicitly explicitly pin long running threads to cpu cores. Traditional database systems typically assign a transaction to a single physical thread. If the transaction blocks, for instance, while waiting for lock acquisition or disk $\mathrm{I} / \mathrm{O}$, the database yields the thread's processor to other threads with non-blocked transactions. In order to adequately utilize processing resources when transactions block, the database ensures that there are a sufficiently large number of threads running other nonblocked transactions. The number of active threads is therefore typically larger than the number of physical processors. In contrast, transactions in single node main memory database systems do not block on I/O. Therefore, some main memory database systems use non-blocking thread implementations such that when a transaction blocks for any reason (such as a a failure to acquire a lock), instead of yielding control to another thread, the thread temporarily stops working on that transaction and picks up another transaction to process, eventually returning to the original transaction when it is no longer blocked [25]. We leverage this approach in our implementations, so that all baselines we experiment with do not need to pay thread context switching costs.

\subsection{Concurrency control scalability}

We begin our experimental evaluation by exploring the effect of the separation concurrency control from transaction execution in BоHм (Section 3). Recall that concurrency control and transaction execution are each handled by two separate modules, each of which is parallelized by a separate group of threads. Both, the number of threads devoted to concurrency control and the number of threads devoted to transaction execution are system parameters that can be varied by a system administrator. We vary both parameters in this experiment.

Our experiment stresses the concurrency control layer as much as possible, in order to test scalability. In particular:

- The workload consists of short, simple transactions, involving only 10 RMWs of different records. Furthermore, each record is very small (it only contains a single 64-bit integer attribute), and the modification that occurs in the transaction consists of a sim- 
ple increment of this integer. As a consequence, the execution time of each transaction's logic is very small.

- The database consists of 1,000,000 records, and the 10 records involved in the RMWs of each transaction are chosen from a uniform distribution. As a consequence, transactions rarely conflict with each other.

- The entire database resides in main memory, so there are no delays to access secondary storage.

As a result of these three characteristics, there are no delays around contending for data, waiting for storage, or executing transaction logic. This stresses the concurrency control layer as much as possible - it is not able to hide behind other bottlenecks and delays in the system, and must keep up with the transaction execution layer, which consumes very little effort in processing each transaction.

Figure 4 shows the results of our experiment. The number of threads devoted to transaction execution is varied on the $\mathrm{x}$-axis, while the number of threads devoted to concurrency control is varied via the 4 separate lines on the graph. Recall that in our experimental setup, there is a 1:1 correspondence between threads and CPU cores. Thus, adding more threads to either concurrency control or transaction execution is equivalent to adding more cores dedicated to these functions.

Despite the extreme stress on the concurrency control layer in this microbenchmark, when the number of concurrency control threads (cores) significantly outnumber the number of execution threads (cores), the system is bottlenecked by transaction execution, not concurrency control. This is why the throughput of each configuration initially increases as more execution threads are added. However, once the throughput of the execution layer matches that of the concurrency control layer, the total throughput plateaus. At this point, the throughput of the system is bottlenecked by the concurrency control layer.

As we increase the number of concurrency control threads (represented by the four separate lines in Figure 4), the maximum throughput of the system increases. This indicates that the concurrency control layer's throughput scales with increasing thread (core) counts. This is because the concurrency control layer is able to exploit greater intra-transaction parallelism and has a lower per-thread cache footprint at higher core counts (Section 3.2.3).

While the number of concurrency control and execution threads can be varied by a system administrator, the choice of the optimal division of threads between the concurrency control and execution layers is non-trivial. As Figure 4 indicates, using too few concurrency control threads results in under utilization of execution threads, while using too many concurrency control threads will constrain overall throughput as not enough execution threads will be available to process transactions.

This problem can be addressed by using techniques for dynamic load balancing in high-performance web-servers. BOHM uses a staged event-driven architecture (SEDA) [32]; the concurrency control and execution phases each correspond to a stage. The processing of a single request (in BoHM's case, a transaction) is divided between the concurrency control and execution phases. As advocated by SEDA, there is a strong separation between the concurrency control and execution phases; threads in the concurrency control phase are unaware of threads in the execution phase (and viceversa). SEDA's design allows for dynamic allocation of threads to stages based on load. By following SEDA's design principles, BOHM can similarly dynamically allocate resources to the concurrency control and execution phases.
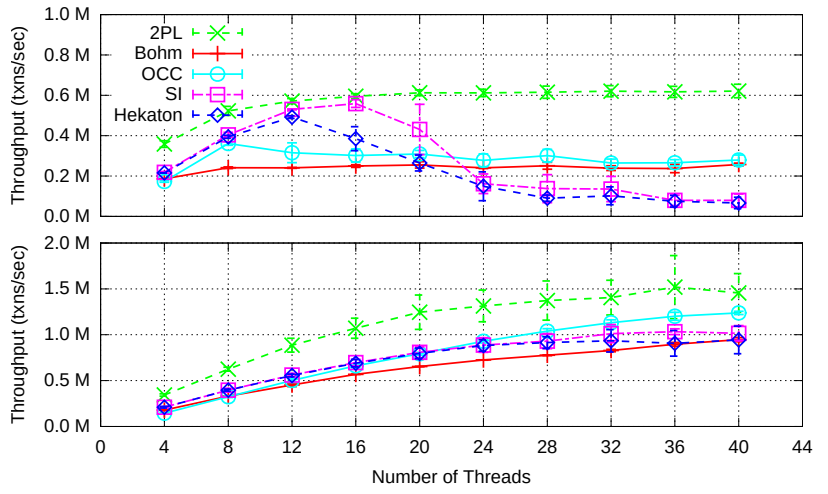

Figure 5: YCSB 10RMW throughput. Top: High Contention $($ theta $=0.9)$. Bottom: Low Contention $($ theta $=0.0)$.

Overall, this initial experiment provides evidence of the scalability of BOHM's design. As we increase the number of concurrency control and execution threads in unison, the overall throughput scales linearly. At its peak in this experiment, BOHM's concurrency control layer is able to handle nearly 2 million transactions a second (which is nearly 20 million RMW operations per second) a number that (to the best of our knowledge) surpasses any known real-world transactional workload that exists today.

\subsection{YCSB}

We now compare BoHM's throughput against the implemented baselines of Hekaton, Snapshot Isolation (SI), OCC, and locking on the Yahoo! Cloud Serving Benchmark (YCSB) [9].

For this set of experiments, we use a single table consisting of $1,000,000$ records, each of size 1,000 bytes (the standard record size in YCSB). We use three kinds of transactions: the first performs 10 read-modify-writes (RMWs) - just like the experiment above, the second performs 2 RMWs and 8 reads (which we call $2 \mathrm{RMW}-8 \mathrm{R}$ ), and the third is a read-only transaction which reads 10,000 records.

We use a workload consisting of only 10RMW transactions to compare the overhead of multiversioning in BOHM compared to a single versioned system. If a workload consists of transactions that perform only RMW operations, we do not expect to obtain any benefits from multiversioning. To understand why, consider two transactions $T_{1}$ and $T_{2}$ whose read- and write-sets consist of a single record, $x$. Since both transactions perform an RMW on $x$, their execution must be serialized. Either $T_{1}$ will observe $T_{2}$ 's write or vice-versa. This serialization is equivalent to how a single version system would handle such a conflict.

In contrast, we expect that, under high contention, multi-versioned systems will execute a workload of $2 \mathrm{RMW}-8 \mathrm{R}$ transactions with greater concurrency than single-versioned systems. The reason is that if a transaction, $T$, only reads the value of record $r$, then $T$ does not need to block a transaction $T^{\prime}$ which writes $r$ (or alternatively, performs an RMW operation on $r$ ).

Finally, we use a workload consisting of a combination of 10RMW and read-only transactions to demonstrate the impact of long running read-only transactions on each of our baselines. We expect such a workload to favor multi-version systems because multi-version systems ensure that read-only transactions execute without blocking conflicting update transactions (and vice-versa).

\subsubsection{RMW Workload}

Our first experiment compares the throughput of each system on YCSB transactions which perform 10RMW operations, where each 
element of a transaction's read- and write-set is unique. We run the experiment under both low and high contention. We use a zipfian distribution to generate the elements in a transaction's read- and write-sets. We vary the contention in the workload by changing the value of the zipfian parameter theta [14]. The low contention experiment sets theta to 0 , while the high contention experiments sets theta to 0.9 .

The graph at the top of Figure 5 shows the result of our experiment under high contention. The throughput of every system does not scale beyond a certain threshold due to the high contention in the workload - there are simply not enough transactions that do not conflict that can be run in parallel. Hekaton and SI perform particularly poorly when there are a large number of concurrently executing threads because under high contention, they are prone to large numbers of aborts. Optimistic systems run transactions concurrently, regardless of the presence of conflicts, and validate that transactions executed in a serializable fashion (or in the case of SI, that write-write conflicts are absent and that transactions read a consistent snapshot of the database). A transaction is aborted if its validation step fails, and the work performed by the transaction is effectively wasted. Note, however, that while OCC is also optimistic and suffers from aborts; it does not suffer from the same drop in throughput as Hekaton and SI. This is because Silo (the version of OCC we use in these experiments) uses a back-off scheme to slow down threads when there is high write-write contention.

The reason why Вонм is outperformed by the locking implementation is that individual transactions are subject to greater overhead. When a multi-version database system performs an RMW operation on a particular record (say, $x$ ), the corresponding execution thread must bring the memory words corresponding to $x$ 's version being read into cache, and write a different set of words corresponding to the new version of $x$. In contrast, when a singleversion system performs an RMW operation, it writes to the same set of memory words it reads. Note that the overhead of creating new versions must be paid during a transaction's contention period $^{3}$. As a consequence, version creation has a greater negative impact on throughput in high contention workloads (as compared to low contention workloads). This effect is magnified for YCSB, since the size of each YCSB record is fairly large (1,000 bytes), and each transaction must therefore pay the overhead of writing ten new 1,000-byte records. Thus, all the multi-versioned systems (including BOHM) have a disadvantage on this workload - they pay the overhead of multi-versioning without getting any benefit of increase in concurrency for this $100 \%$ RMW benchmark.

Nonetheless, BoHM's lack of aborts allow it to achieve over twice the throughput of the other multi-versioned systems (Hekaton and SI) when there are large numbers of concurrently running threads. However, when there are low numbers of concurrently running threads, there is less contention and the optimistic systems do not suffer from many aborts. Furthermore, the high theta increases the number of versions created and ultimately garbage collected for the "hot" records, and our configuration of Hekaton and SI to not have to garbage collect give them a small advantage over BoHM.

We find that OCC's throughput begins to degrade between 8 and 12 threads, while Hekaton and SI are able to sustain higher throughput for slightly higher thread counts (12 and 16 threads respectively). The reason for this is that Hekaton and SI use an optimization that allows transactions to speculatively read uncommitted values (commit dependencies) [19].

\footnotetext{
${ }^{3}$ We define a transaction's contention period as the time period during which concurrently running conflicting transactions must either block or abort (depending on the pessimistic or optimistic nature of the concurrency control protocol).
}
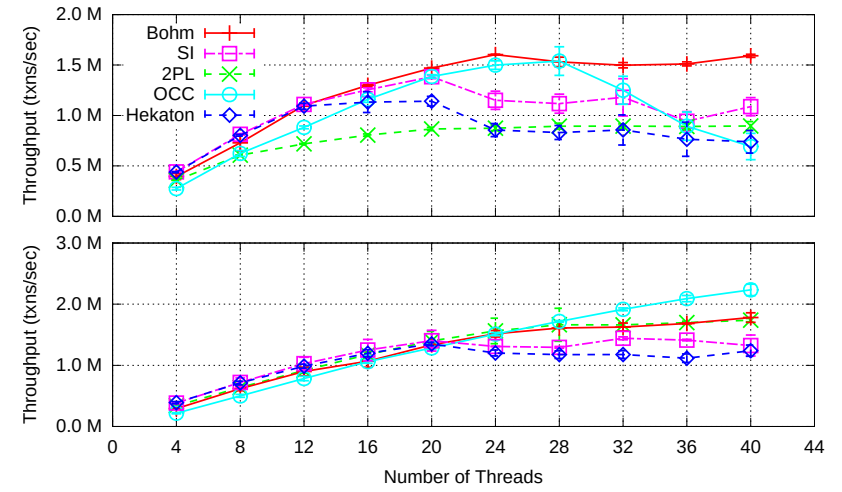

Figure 6: YCSB 2RMW-8R throughput. Top: High Contention $($ Theta $=0.9)$. Bottom: Low Contention $($ Theta $=0.0)$.

The graph at the bottom of Figure 5 shows the same experiment under low contention. We find that locking once again outperforms the other concurrency control protocols; however, the difference is much smaller. The reason why locking still outperforms OCC is that most OCC implementations (including our implementation of Silo OCC [31]) requires threads to buffer their writes locally prior to making writes visible in the database. Locking does not pay the overhead of copying buffered writes to database records. While OCC's write buffering is similar to the multi-version systems's requirement of creation of new versions, it has lower overhead because the same local write buffer can be re-used by a single execution thread across many different transactions (leading to better cache locality of the local write buffers). In contrast, the multiversion systems need to write different locations on every update.

Under low contention, the multi-version systems - BoHM, Hekaton, and SI - have similar performance. Hekaton and SI marginally outperform BOHM, since our implementations of Hekaton and SI do not include garbage collection and use array-based indices to access records.

\subsubsection{RMW-8R Workload}

This section compares BoHM's throughput with each of our baselines on a workload where each YCSB transaction performs two RMWs and eight reads (2RMW-8R). In a high contention setting, we expect that the multi-versioned systems will obtain more concurrency than the single-versioned systems, since the reads and writes of the same data items need not conflict under certain circumstances. In particular, under SI, reads and writes never conflict. Therefore, it is theoretically able to achieve more concurrency than any of the other systems which guarantee serializability and therefore have to restrict (to some degree), reads and writes of the same data items in order to avoid the write-skew anomaly (see Section 2). In particular, BOHM allows writes to block reads, but reads never block writes. In Hekaton, reads also never block writes, but writes can cause transactions that read the same data items to abort. In the single-version systems, reads and writes always conflict either via blocking (2PL) or aborting (OCC).

The graph at the top of Figure 6 shows the results of this experiment under high contention. As expected, the multi-versioned implementations outperform the single-versioned implementations due to their ability to achieve higher concurrency, and SI outperforms most of the other systems due to the larger amount of concurrency possible when serializable isolation is not required.

Surprisingly, however, BOHM significantly outperforms SI. We attribute this difference to aborts induced by write-write conflicts in SI. Under high contention this can lead to many aborts and wasted 


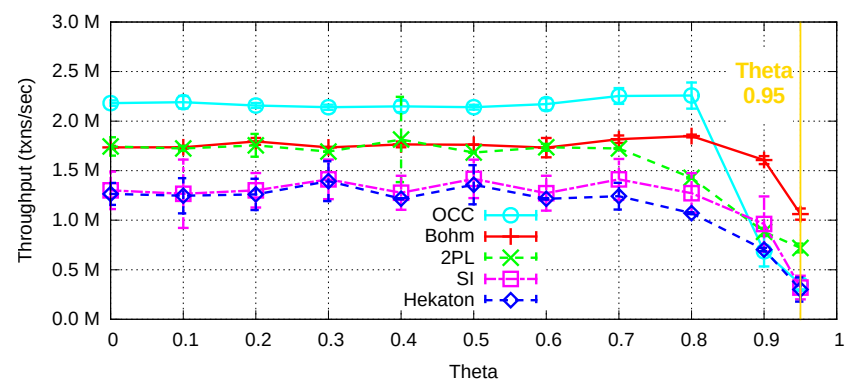

Figure 7: YCSB 2RMW-8R throughput varying contention

work. Meanwhile, BоHм specifies the correct ordering of writes to the same record across transactions in the concurrency control layer, so that the transaction processing layer simply needs to fill in placeholders and never needs to abort transactions due to writewrite conflicts (Section 3.3.1). Like SI, Hekaton also suffers from aborts and wasted work under high contention. Interestingly, the Hekaton paper also implements a pessimistic version of its concurrency control protocol, but finds that it performs worse than the optimistic version, even under high contention. This is because in the pessimistic version, reads acquire read locks, and thus conflict with writes to the same record, thereby reducing concurrency. Thus, a major contribution of BoHM relative to Hekaton is a solution for allowing reads to avoid blocking writes without resorting to optimistic mechanisms.

The graph at the bottom of Figure 6 shows the same experiment under low contention. OCC outperforms both BOHM and locking as it employs a light-weight concurrency control protocol, and does not suffer from aborts under low contention. In particular, this workload contains a significant number of reads, and read validation is very cheap in Silo (which is our OCC implementation) [31]. Note however, that BOHM is very close in performance to OCC, despite the additional overhead of maintaining multiple versions.

The slope of the OCC, locking, and BOHM lines all decrease at higher thread counts. We attribute this to the fact that our database tables span multiple NUMA sockets.

The most interesting part of the bottom of Figure 6 is the comparison of the three multi-versioning implementations. With no contention, there are very few aborts in optimistic schemes, nor any significant differences between the amount of concurrency between the three schemes. Therefore, one might expect all three implementations to perform the same. However, we find that this is not the case; Hekaton and SI are unable to scale beyond 20 cores. We attribute Hekaton and SI's poor performance to contention on global transaction timestamp counter. Hekaton and SI use a global 64bit counter to assign transactions their begin and end timestamps. In order to obtain a timestamp, both systems atomically increment the value of the counter using an atomic fetch-and-increment instruction (xaddq on our x86-64 machine). The counter is incremented at least twice for every transaction, regardless of the presence of actual conflicts ${ }^{4}$. At high thread counts, SI and Hekaton are bottlenecked by contention on this global counter. This observation is significant because it indicates that database designs which rely on centralized contended data-structures are fundamentally unscalable. BOHM's avoidance of this prevalent limitation of multi-version concurrency control protocols is thus an important contribution.

Figure 7 further illustrates the fundamental issue with Hekaton and SI's inability to scale. The graph shows the throughput of each

\footnotetext{
${ }^{4}$ The counter may be incremented more than twice if a transaction needs to be re-executed due to a concurrency control induced abort.
}

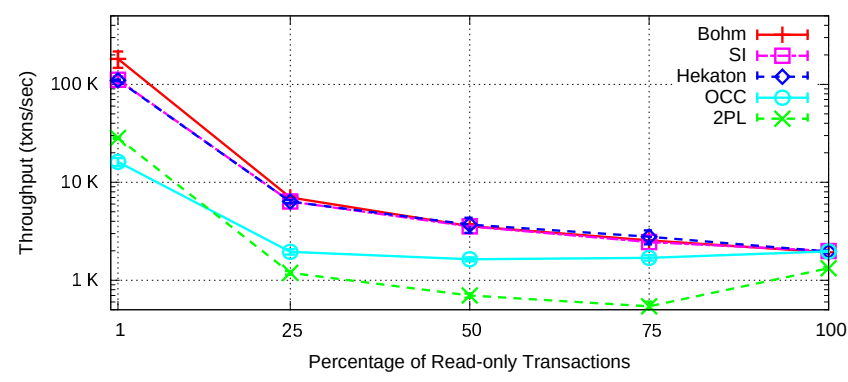

Figure 8: YCSB throughput with long running read-only transactions.

system (at 40 threads) while varying the degree of contention in the workload. We use the same 2RMW-8R workload. The graph indicates that both Hekaton and SI have identical performance under low to medium contention, as they are both limited by the timestamp counter bottleneck. Only under high contention does a new bottleneck appear, and prevents the timestamp counter from being the primary limitation of performance.

\subsubsection{Impact of Long Read-only Transactions}

In this section, we measure the effect of long running read-only transactions on each of our baselines. We run each baseline on a workload consisting of a mix of update and read-only transactions. Update transactions are the low contention 10RMW YCSB transactions from Section 4.2.1. Read-only transactions read 10,000 records - chosen uniformly at random - from the database.

Figure 8 plots the overall throughput of each system while varying the fraction of read-only transactions in the workload. When a small fraction of the transactions are read-only (1\%), we find that the multi-version systems outperform OCC and locking by about an order of magnitude (the y-axis uses a log-scale). This is because single-version systems cannot overlap the execution of read-only transactions and update transactions. In the multi-versioned systems, read-only transactions do not block the execution of conflicting update transactions (and vice-versa) because read-only transactions can perform their reads as of a timestamp which precedes the earliest active update transaction. We also find that BOHM significantly outperforms Hekaton and SI. We attribute this difference to BoHM's read-set optimization (Section 3.2.3), which ensures that BоHм can obtain a reference to the version of a record required by a transaction without accessing any preceding or succeeding versions. In contrast, in Hekaton and SI, if the version required by a transaction, $v_{i}$, has been overwritten, then the system must traverse the list of succeeding versions $v_{n}, v_{n-1}, \ldots, v_{i+1}$ (where $n>i$ ) in order to obtain a reference to $v_{i}$. Version traversal overhead is not specific to our implementations of Hekaton and SI - it is inherent in systems which determine the visibility of each transaction's writes after the transaction has finished executing. Thus, version traversal overhead is unavoidable in all conventional multi-version systems.

As the fraction of read-only transactions increases, the throughput of each system drops. This is because each read-only transaction runs for a significantly greater duration than update transactions (read-only transactions read 10,000 records, while update transactions perform an RMW operation on 10 records). When the workload consists of $100 \%$ read-only transactions, all systems exhibit nearly identical performance. This is because the workload does not contain any read-write nor write-write conflicts.

\subsection{SmallBank Benchmark}

Our final set of experiments evaluate BoHM's performance on the SmallBank benchmark [7]. This benchmark was used by Cahill 

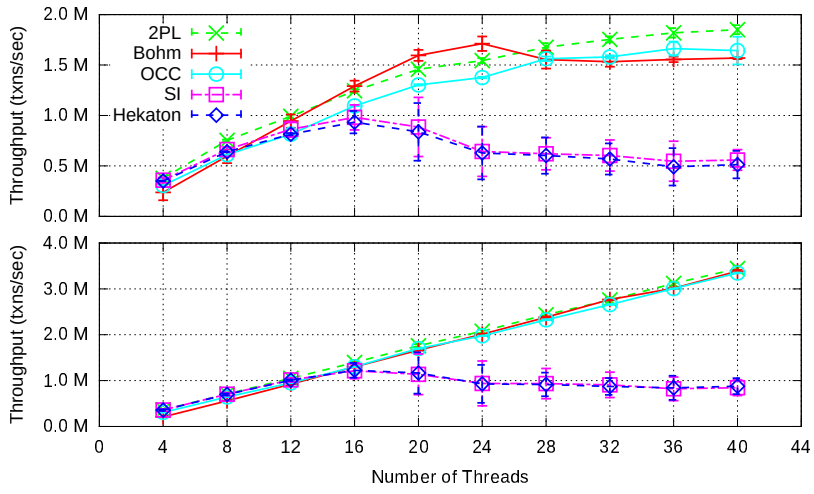

Figure 9: Small Bank throughput. Top: High Contention (50 Customers). Bottom: Low Contention (100,000 Customers).

et al. for their research on serializable multi-versioned concurrency control. SmallBank is designed to simulate a banking application. The application consists of three tables, (1) Customer, a table which maps a customer's name to a customer identifier, (2) Savings, a table whose rows contain tuples of the form $<$ Customer Identifier, Balance $>$, (3) Checking, a table whose rows contain tuples of the form $<$ Customer Identifier, Balance $>$. The application consists of five transactions: (1) Balance, a read-only transaction which reads a single customer's checking and savings balances, (2) Deposit, makes a deposit into a customer's checking account, (3) TransactSaving, makes a deposit or withdrawal on a customer's savings account, (4) Amalgamate, moves all funds from one customer to another, (5) WriteCheck, which writes a check against an account. None of the transactions update the customer table only the Savings and Checking tables are updated.

The number of rows in the Savings and Checking tables is equal to the number of customers in the SmallBank database. We can therefore vary the degree of contention in our experiments by changing the number of customers; decreasing the number of customers increases the degree of contention in the SmallBank workload.

The transactions in the SmallBank workload are much smaller than the transactions in the YCSB workload from the previous section. Every transaction performs reads and writes on between 1 and 3 rows. Each record in the Savings and Checking tables is 8 bytes long. In comparison, our configuration of the YCSB workload performs exactly 10 operations on each transaction, and each record is of size 1,000 bytes. In order to make the SmallBank transactions slightly less trivial in size, each transaction spins for 50 microseconds (in addition to performing the logic of the transaction).

Figure 9 shows the results of our experiment. The graph at the top of Figure 9 shows the results under high contention (the number of SmallBank customers is set to 50). Although locking once again performs best under high contention, the difference between locking and BOHM is not as large as in the contended 10RMW YCSB experiment (Section 4.2.1). There are two reasons for this difference:

First, as explained in Section 4.2.1, BoHM must pay the cost of bringing two different sets of memory words into cache on a read-modify-write operation, one corresponding to the version that needs to be read, the second corresponding to the version to be created. Since SmallBank's 8-byte records are smaller than YCSB's 1000-byte records, the cost of this extra memory access is smaller. Hence, the relative difference between BOHM and locking is smaller.
Second, the workload from Section 4.2.1 was 100\% RMW transactions. In contrast, a small part of the SmallBank workload (20\% of all transactions) consist of read-only Balance transactions. Multiversioned approaches such as ВОНм are thus able to increase the concurrency of these transactions, since reads do not block writes.

Both Hekaton and SI's throughput drop under high contention due to concurrency control induced aborts. At 40 threads, SI outperforms Hekaton by about 50,000 transactions per second because it suffers from fewer aborts while validating transactions. Note that the abort-related drop in performance of Hekaton and SI is greater than OCC. This is because the contention on the timestamp counter for the multi-versioned schemes (Hekaton and SI) increases the time required to get a timestamp. Since the SmallBank transactions are so short, this time to acquire a timestamp is a nontrivial percentage of overall transaction length. Hence, the transactions are effectively longer for Hekaton and SI than they are for OCC, which leads to more conflict during validation, and ultimately more aborts.

The graph at the bottom of Figure 9 shows the results of the same experiment under low contention. We find that locking, OCC, and BOHM have similar performance under this configuration. As mentioned previously, the cost of RMW operations on SmallBank's 8-byte records is much smaller than RMW operations on YCSB's 1000-byte records.

As we saw in previous experiments (Section 4.2.2), we find that both Hekaton and SI are bottlenecked by contention on the global timestamp counter. When using 40 threads, BoHM is able to achieve throughput in excess of 3 million transactions per second, while Hekaton and SI achieve about 1 million transactions per second; a difference of more than $3 x$.

\section{RELATED WORK}

Multi-core Scalability. Pandis et al. propose a data-oriented architecture (DORA) in order to eliminate the impact of contended accesses to shared memory by transaction execution threads [24]. DORA partitions a database among several physical cores of a multi-core system and executes a disjoint subset of each transaction's logic on multiple threads, a form of intra-transaction parallelism. BоHм uses intra-transaction parallelism to decide the order in which transactions must execute. However, the execution of a transaction's logic occurs on a single thread.

Jung et al. propose techniques for improving the scalability of lock-managers [18]. Their design includes the pervasive use of the read-after-write pattern [5] in order to avoid repeatedly "bouncing" cache-lines due to cache-coherence $[4,22]$. In addition, to avoid the cost of reference counting locks, they use a technique to lazily de-allocate locks in batches. BOHM similarly refrains from the use of reference counters to garbage collect versions of records that are no longer visible to transactions.

Johnson et al. identified latch contention on high level intention locks as a scalability bottleneck in multi-core databases [17]. They proposed Speculative Lock Inheritance (SLI), a technique to reduce the number of contended latch acquisitions. SLI effectively amortizes the cost of contended latch acquisitions across a batch transactions by passing hot locks from transaction to transaction without requiring calls to the lock manager. BOHM similarly amortizes synchronization across batches of transactions in order to scale concurrency control.

Very lightweight locking (VLL) reduces lock-manager overhead by co-locating concurrency control related meta-data with records [25]. Unlike BoHM, VLL is not designed for systems with large number of cores because every transaction must execute a global critical section before it can execute. 
Calvin [29] is a deterministic database system that executes transactions according to a pre-defined total order. Calvin uses deterministic transaction ordering to reduce the impact of distributed transactions on scalability. Furthermore, Calvin uses a modular architecture and separates key parts of concurrency control from transaction execution [28]. Although similar to BOHM with its focus on scalability and modularity, Calvin is a single-versioned system and uses locking to avoid read-write and write-write conflicts, while BOHM is multi-versioned and ensures that reads do not block writes. Furthermore, Calvin is focused on horizontal shared-nothing scalability, while Вонм is focused on multi-core scalability.

H-Store [27] uses a shared-nothing architecture consisting of single-threaded partitions in order reduce the impact of lock-manager overhead [15], and logging overhead [20]. However, performance degrades rapidly if a workload contains multi-partition transactions. Furthermore, sub-optimal performance is observed if some partitions have more work to do than others. BOHM achieves scalability without doing a hard-partitioning of the data - it is thus less susceptible to skew problems and does not suffer from the multipartition transaction problem.

Dependency Graphs. Whitney et al. propose a deterministic concurrency control in which transactions are executed according to a pre-defined total order [33]. Their system derives concurrency by constructing a graph of transactions, which defines a partial order on transactions based on their conflicts. BOHM also pre-defines the order in which transactions must execute, but its design is fundamentally motivated by multi-core scalability. In contrast, Whitney et al. 's system contains several centralized bottlenecks which inhibit multi-core scalability (e.g., the set of transactions that are ready to execute is maintained in a centralized data-structure).

Faleiro et al. describe a technique for lazily evaluating transactions in the context of deterministic database systems [10]. This lazy database design separates concurrency control from transaction execution - a design element that is shared by BoHM. However, Вонм does not process transactions lazily, and is far more scalable due to its use of intra-transaction parallelism, and avoiding writes to shared memory on reads. Furthermore, BOHM is designed to be a generic multi-versioned concurrency control technique, and is motivated by existing limitations in multi-version concurrency control systems.

\section{CONCLUSIONS}

Most multi-versioned database systems either do not guarantee serializability or only do so at the expense of significant reductions in read-write concurrency. In contrast BOHM is able to achieve serializable concurrency control while still leveraging the multiple versions to ensure that reads do not block writes. Our experiments have shown that this enables BOHM to significantly outperform other multi-versioned systems. Further, for workloads where multi-versioning is particularly helpful (workloads containing a mixture of reads and writes at high contention), BоHM is able to outperform both single-versioned optimistic and pessimistic systems, without giving up serializability. BOHM is the first multiversioned database system to accomplish this in main-memory multicore environments.

Acknowledgments This work was sponsored by the NSF under grant IIS-1249722 and by a Sloan Research Fellowship. We thank Phil Bernstein, Alexander Thomson, and the anonymous VLDB 2015 reviewers for their insightful feedback on earlier drafts of this paper.

\section{REFERENCES}

[1] A. Adya, B. Liskov, and P. O'Neil. Generalized isolation level definitions. In Proc. of ICDE, pages 67-78. IEEE, 2000.

[2] D. Agrawal, A. J. Bernstein, P. Gupta, and S. Sengupta. Distributed optimistic concurrency control with reduced rollback. Distributed Computing, 2(1):45-59, 1987.

[3] M. K. Aguilera, A. Merchant, M. Shah, A. Veitch, and C. Karamanolis. Sinfonia: a new paradigm for building scalable distributed systems. In ACM SIGOPS Operating Systems Review, volume 41, pages 159-174. ACM, 2007.

[4] T. E. Anderson. The performance of spin lock alternatives for shared-money multiprocessors. IEEE TPDS, 1(1):6-16, 1990.

[5] H. Attiya, R. Guerraoui, D. Hendler, P. Kuznetsov, M. M. Michael, and $\mathrm{M}$. Vechev. Laws of order: expensive synchronization in concurrent algorithms cannot be eliminated. In ACM SIGPLAN Notices, volume 46, 2011.

[6] H. Berenson, P. Bernstein, J. Gray, J. Melton, E. O'Neil, and P. O'Neil. A critique of ansi sql isolation levels. In Proc. of SIGMOD, pages 1-10, 1995.

[7] M. J. Cahill. Serializable Isolation for Snapshot Databases. $\mathrm{PhD}$ thesis, University of Sydney, 2009.

[8] M. J. Cahill, U. Röhm, and A. D. Fekete. Serializable isolation for snapshot databases. In Proc. of SIGMOD, pages 729-738, 2008.

[9] B. F. Cooper, A. Silberstein, E. Tam, R. Ramakrishnan, and R. Sears. Benchmarking cloud serving systems with ycsb. SoCC, 2010.

[10] J. M. Faleiro, A. Thomson, and D. J. Abadi. Lazy evaluation of transactions in database systems. Proc. of SIGMOD, pages 15-26, 2014.

[11] A. Fekete. Allocating isolation levels to transactions. In Proc. of PODS, 2005.

[12] A. Fekete, D. Liarokapis, E. O'Neil, P. O’Neil, and D. Shasha. Making snapshot isolation serializable. TODS, 30(2):492-528, 2005.

[13] A. Fekete, E. O'Neil, and P. O'Neil. A read-only transaction anomaly under snapshot isolation. ACM SIGMOD Record, 33(3):12-14, 2004.

[14] J. Gray, P. Sundaresan, S. Englert, K. Baclawski, and P. J. Weinberger. Quickly generating billion-record synthetic databases. Proc. of SIGMOD, 1994.

[15] S. Harizopoulos, D. Abadi, S. Madden, and M. Stonebraker. Oltp through the looking glass, and what we found there. Proc. of SIGMOD, 2008.

[16] K. Jacobs, R. Bamford, G. Doherty, K. Haas, M. Holt, F. Putzolu, and B. Quigley. Concurrency control, transaction isolation, and serializability in SQL 92 and Oracle 7. Oracle Whitepaper, Part No. A33745, 1995.

[17] R. Johnson, I. Pandis, and A. Ailamaki. Improving oltp scalability using speculative lock inheritance. PVLDB, 2(1):479-489, 2009.

[18] H. Jung, H. Han, A. D. Fekete, G. Heiser, and H. Y. Yeom. A scalable lock manager for multicores. In Proc. of SIGMOD, 2013.

[19] P.-A. Larson, S. Blanas, C. Diaconu, C. Freedman, J. M. Patel, and M. Zwilling. High-performance concurrency control mechanisms for main-memory databases. PVLDB, 5(4):298-309, Dec. 2011.

[20] N. Malviya, A. Weisberg, S. Madden, and M. Stonebraker. Rethinking main memory oltp recovery. In Proc. of ICDE, 2014.

[21] P. E. Mckenney, J. Appavoo, A. Kleen, O. Krieger, O. Krieger, R. Russell, D. Sarma, and M. Soni. Read-copy update. In In Ottawa Linux Symposium, pages 338-367, 2001

[22] J. M. Mellor-Crummey and M. L. Scott. Algorithms for scalable synchronization on shared-memory multiprocessors. ACM Transactions on Computer Systems (TOCS), 9(1):21-65, 1991.

[23] I. Moraru, D. G. Andersen, and M. Kaminsky. There is more consensus in egalitarian parliaments. In Proc. of SOSP, 2013.

[24] I. Pandis, R. Johnson, N. Hardavellas, and A. Ailamaki. Data-oriented transaction execution. PVLDB, 3(1-2):928-939, 2010.

[25] K. Ren, A. Thomson, and D. J. Abadi. Lightweight locking for main memory database systems. PVLDB, 6(2):145-156, Dec. 2012.

[26] K. Ren, A. Thomson, and D. J. Abadi. An evaluation of the advantages and disadvantages of deterministic database systems. PVLDB, 7(10):821-832, 2014.

[27] M. Stonebraker, S. R. Madden, D. J. Abadi, S. Harizopoulos, N. Hachem, and P. Helland. The end of an architectural era (it's time for a complete rewrite). In Proc. of VLDB, 2007.

[28] A. Thomson and D. J. Abadi. Modularity and scalability in calvin. IEEE Data Engineering Bulletin, 36(2): 48-55, 2013.

[29] A. Thomson, T. Diamond, S. chun Weng, K. Ren, P. Shao, and D. J. Abadi. Calvin: Fast distributed transactions for partitioned database systems. In SIGMOD, 2012.

[30] A. Thomson, T. Diamond, S.-C. Weng, K. Ren, P. Shao, and D. J. Abadi. Fast distributed transactions and strongly consistent replication for oltp database systems. ACM Trans. Database Syst., 39(2):11:1-11:39, May 2014.

[31] S. Tu, W. Zheng, E. Kohler, B. Liskov, and S. Madden. Speedy transactions in multicore in-memory databases. SOSP, 2013.

[32] M. Welsh, D. Culler, and E. Brewer. Seda: An architecture for well-conditioned, scalable internet services. In SOSP, 2001.

[33] A. Whitney, D. Shasha, and S. Apter. High volume transaction processing without concurrency control, two phase commit, SQL or C++. In HPTS, 1997. 